\title{
Completion of the Wilf-Classification of 3-5 Pairs Using Generating Trees
}

\author{
Mark Lipson * \\ Harvard University \\ Department of Mathematics \\ Cambridge, MA 02138 \\ mark.lipson@gmail.com
}

Submitted: Jan 31, 2006; Accepted: Mar 15, 2006; Published: Apr 4, 2006

Mathematics Subject Classifications: 05A05, 05A15

\begin{abstract}
A permutation $\pi$ is said to avoid the permutation $\tau$ if no subsequence in $\pi$ has the same order relations as $\tau$. Two sets of permutations $\Pi_{1}$ and $\Pi_{2}$ are Wilfequivalent if, for all $n$, the number of permutations of length $n$ avoiding all of the permutations in $\Pi_{1}$ equals the number of permutations of length $n$ avoiding all of the permutations in $\Pi_{2}$. Using generating trees, we complete the problem of finding all Wilf-equivalences among pairs of permutations of which one has length 3 and the other has length 5 by proving that $\{123,32541\}$ is Wilf-equivalent to $\{123,43251\}$ and that $\{123,42513\}$ is Wilf-equivalent to $\{132,34215\}$. In addition, we provide generating trees for fourteen other pairs, among which there are two examples of pairs that give rise to isomorphic generating trees.
\end{abstract}

\section{Introduction}

\subsection{Pattern Avoidance and Wilf-Equivalence}

We denote a permutation $\pi$ of the numbers $\{1,2, \ldots, n\}$ by $\pi=\pi_{1} \pi_{2} \cdots \pi_{n}$, where $\pi_{i}=$ $\pi(i)$ for $1 \leq i \leq n$. For permutations $\pi=\pi_{1} \pi_{2} \cdots \pi_{n}$ and $\tau=\tau_{1} \tau_{2} \cdots \tau_{m}$, we say that $\pi$ contains $\tau$ if there exist indices $1 \leq i_{1}<i_{2}<\cdots<i_{m} \leq n$ such that $\pi_{i_{k}}<\pi_{i_{l}}$ if and only if $\tau_{k}<\tau_{l}$. If no such indices exist, then we say that $\pi$ avoids $\tau$.

For a set of permutations $\Pi$, we let $S_{n}(\Pi)$ denote the set of permutations of length $n$ avoiding all of the permutations $\tau \in \Pi$, and we let $s_{n}(\Pi)$ denote the cardinality of $S_{n}(\Pi)$. Two sets $\Pi$ and $\Sigma$ are said to be Wilf-equivalent if $s_{n}(\Pi)=s_{n}(\Sigma)$ for all $n$.

${ }^{*}$ Please send correspondance to the following address: 9 Sheridan St., Lexington, MA 02420 
Wilf-equivalence defines an equivalence relation on sets of permutations, and we call the resulting equivalence classes Wilf-classes.

The problem of counting the permutations avoiding a given permutation or set of permutations is a rich one. One of the oldest and most famous results in the area is a theorem of Erdös and Szekeres [3], which states that $s_{n}(12 \cdots k,(l)(l-1) \cdots 1)=0$ for $n>(k-1)(l-1)$. The field has experienced rapid growth in the last twenty years, beginning with Simion and Schmidt's proof that $\{123\}$ and $\{132\}$ are Wilf-equivalent [14]. Since then, all permutations of length 7 and less have been Wilf-classified (see [15]), as well as all sets of two permutations both of length 4 or less (see [6], [14], and [18]). In 2004, Marcus and Tardos proved the Stanley-Wilf conjecture, which states that for any set $\Pi, s_{n}(\Pi)$ grows at most exponentially in $n$ [13]. The study of permutation avoidance has also found applications to a variety of other problems in combinatorics, as well as areas of algebraic geometry and computer science (see [4] and [15]).

\subsection{Overview of Results}

In Sections 2.1 and 2.2, we prove the two Wilf-equivalences

$$
s_{n}(123,32541)=s_{n}(123,43251) \text { and } s_{n}(123,42513)=s_{n}(132,34215),
$$

completing the Wilf-classification of all pairs of permutations having lengths 3 and 5 (referred to as 3-5 pairs). These results have been derived independently by Mansour, who currently has no plans to publish them (from personal communication, [8]).

Combined with previous results (see [7], [10], and [12]), we find that there are seven Wilf-classes of 3-5 pairs containing at least two pairs that are non-trivially Wilf-equivalent (that is, not by symmetry; see Section 1.4). Of the seven Wilf-classes, the largest is the class of pairs $\Pi$ such that $s_{n}(\Pi)=\left(3^{n-1}+1\right) / 2$, which contains a total of twenty-nine pairs (including some that are Wilf-equivalent by symmetry; see [7] and [10]). In Section 3.1, we give (without complete proofs) the generating trees for fourteen 3-5 pairs that have already been proven to belong to this large class. We find two instances in which two 3-5 pairs give rise to isomorphic trees, a stronger equivalence than Wilf-equivalence.

Finally, we include an example of a generating tree for a 3-6 pair in Section 3.2 and we conclude with a discussion of a few ideas for related open problems in Section 4.

\subsection{Definitions and Conventions}

In figures depicting permutations, $\pi_{i}$ will be to the left of $\pi_{j}$ if $i<j$ and $\pi_{i}$ will be higher than $\pi_{j}$ if $\pi_{i}>\pi_{j}$. The $\pi_{i}$ will often be referred to as elements of the permutation.

If $\pi$ contains $\tau$, with $\pi_{i_{1}} \pi_{i_{2}} \cdots \pi_{i_{m}}$ having the same order relations as $\tau$, then we say that $\pi_{i_{1}} \pi_{i_{2}} \cdots \pi_{i_{m}}$ is a subsequence of $\pi$ of type $\tau$ and that $\pi_{i_{1}} \pi_{i_{2}} \cdots \pi_{i_{m}}$ is an occurrence of $\tau$ in $\pi$. We will often say that $\pi_{i_{k}}$ plays the $\tau_{k}$ in the subsequence of type $\tau$.

We will refer to permutations $\tau$ as patterns in the context of being contained in or avoided by longer permutations $\pi$.

By convention, $s_{0}(\Pi)=1$ for any set $\Pi$. 


\subsection{Symmetries}

Given a permutation $\pi=\pi_{1} \pi_{2} \cdots \pi_{k}$, we define the following three operations:

The reverse of $\pi$ is $\pi_{k} \pi_{k-1} \cdots \pi_{1}$.

The complement of $\pi$ is $\left(k+1-\pi_{1}\right)\left(k+1-\pi_{2}\right) \cdots\left(k+1-\pi_{k}\right)$.

The inverse of $\pi$ is $\pi^{-1}(1) \pi^{-1}(2) \cdots \pi^{-1}(k)$.

If we view permutations as matrices, then $\pi$ avoids the pattern $\tau$ if and only if the permutation matrix for $\pi$ does not contain the matrix for $\tau$ as a minor. Note that the three operations defined above correspond to reflections of permutation matrices about vertical, horizontal, and upper-left-to-lower-right-diagonal axes. By symmetry, then, it is clear that $\pi$ avoids the set of patterns $\Gamma$ if and only if $f(\pi)$ avoids the set $\{f(\gamma) \mid \gamma \in \Gamma\}$, where $f$ is any composition of the reversal, complementation, and inversion operations. Thus, sets of the form $\Gamma$ and $\{f(\gamma) \mid \gamma \in \Gamma\}$ are trivially Wilf-equivalent.

\subsection{3-5 Pairs}

The symmetry arguments in Section 1.4 considerably reduce the problem of determining the Wilf-equivalences among all 720 3-5 pairs. Any 3-5 pair is trivially Wilf-equivalent to a pair of the form $\{123, \tau\}$ or $\{132, \tau\}$ for some $\tau$ of length 5 , so we may restrict our attention to these 240 pairs. Also, if $\tau$ contains 123, for example, then $S_{n}(123, \tau)=S_{n}(123)$, because any permutation that avoids 123 also avoids $\tau$. There are forty-two permutations of length 5 that avoid 123 and forty-two that avoid 132, so we need only consider the corresponding eighty-four pairs. Finally, these pairs can be divided into forty-two Wilf-classes by further symmetry arguments; for example, $s_{n}(123,43251)=s_{n}(123,53214)$ because 123 is the inverse of 123 and 53214 is the inverse of 43251.

In [10], Mansour and Vainshtein derive the generating function

$$
\sum_{n=0}^{\infty} s_{n}(132, \tau) x^{n}
$$

for any pattern $\tau$ avoiding 132. For $\tau$ of length 5, their results lead to the following nontrivial Wilf-equivalences:

$$
\begin{aligned}
s_{n}(132,12345) & =s_{n}(132,21345)=s_{n}(132,23145)=s_{n}(132,23415) \\
& =s_{n}(132,23451)=s_{n}(132,32415)=s_{n}(132,32451) \\
& =s_{n}(132,34125)=s_{n}(132,34251)=s_{n}(132,34512) \\
& =s_{n}(132,42351)=s_{n}(132,43512)=\frac{3^{n-1}+1}{2} ;
\end{aligned}
$$




$$
\begin{aligned}
s_{n}(132,34521)= & s_{n}(132,43521)=s_{n}(132,52341)=s_{n}(132,53241) ; \\
& s_{n}(132,34215)=s_{n}(132,42315) ; \\
& s_{n}(132,32145)=s_{n}(132,43251) ; \\
& s_{n}(132,45231)=s_{n}(132,45312) ; \text { and } \\
& s_{n}(132,45321)=s_{n}(132,53421) .
\end{aligned}
$$

It is easy to verify by computation that these pairs belong to six distinct Wilf-classes.

Before this paper, the only non-trivial Wilf-equivalence known (see [7], [9], [11], [12]) for $3-5$ pairs of the form $\{123, \tau\}$ was

$$
s_{n}(123,15432)=s_{n}(123,21543)=s_{n}(123,32514)=\frac{3^{n-1}+1}{2} .
$$

By computing $s_{10}$, we find that the only other possible Wilf-equivalences among 3-5 pairs are

$$
s_{n}(123,32541)=s_{n}(123,43251) \text { and } s_{n}(123,42513)=s_{n}(132,34215) .
$$

The generating functions $\sum_{n=0}^{\infty} s_{n} x^{n}$ are already known for the pairs $\{123,43251\}$ and $\{132,34215\}([16]$ and $[10])$.

\subsection{Generating Trees}

The most important tools we will use to study 3-5 pairs are generating trees. A generating tree is a rooted, labeled tree, together with a set of rules, called the succession rules of the tree, that uniquely specify the number and labels of the children of any node given its label. A tree is often specified by its root and succession rules, as in this example [18], having a single rule:

$$
\begin{array}{ll}
\text { Root: } & (1) \\
\text { Rule: } & (k) \rightarrow(k+1)(1)^{k-1} .
\end{array}
$$

In this generating tree, a node with label $(k)$ has $k$ children, one labeled $(k+1)$ and $k-1$ labeled (1). We will divide our trees into rows, with the root-node in row 1 , its children in row 2 , and so on. It is easy to see that the tree above has $2^{n-2}$ nodes in row $n$ for $n>1$. The first four rows are shown in Figure 1.

Two trees having the same root and the same succession rules are said to be isomorphic.

Generating trees are useful in many counting problems. They were first used by Chung et al. in [2] to count Baxter permutations and have been applied to the study of patternavoiding permutations on numerous occasions (see, for example, [1], [5], [16], [17], and $[18])$.

In the context of pattern avoidance, the nodes in a generating tree correspond to the permutations avoiding a certain set of patterns $\Pi$, with permutations of length $m$ corresponding to the nodes in row $m$. The root, in particular, always corresponds to the length-1 permutation. Succession rules are derived by considering the active sites 


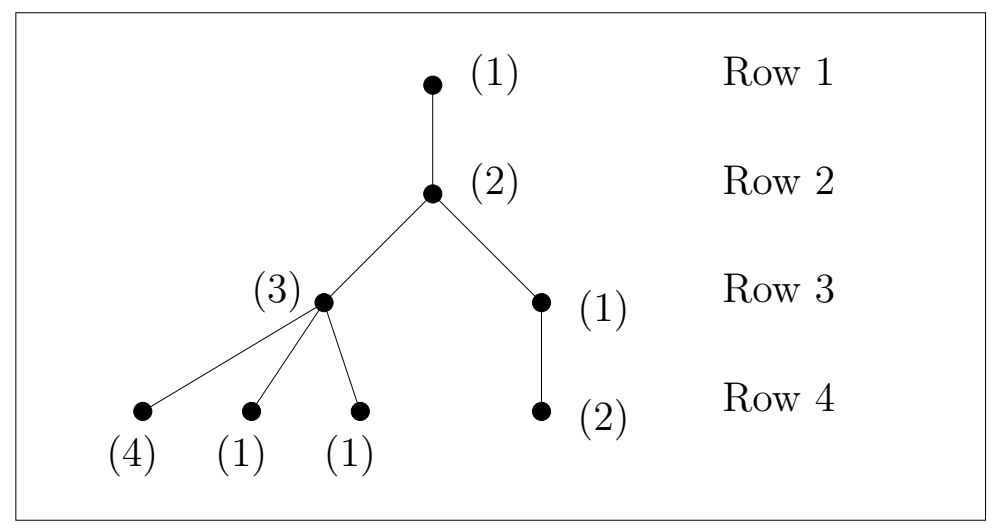

Figure 1: A generating tree with $2^{n-2}$ nodes in row $n$ for $n>1$. The root of the tree is $(1)$, and the succession rule is $(k) \rightarrow(k+1)(1)^{k-1}$.

of a permutation, the spaces between two adjacent elements where we may insert a new, largest element in order to create a permutation that is one element longer and still avoids П. The term child will be used to refer both to a new permutation formed in this way and to the node in the tree corresponding to it.

Thus, a node corresponding to a permutatation $\pi$ of length $n-1$ with $k$ active sites will have $k$ children in a generating tree. These $k$ nodes will correspond to the permutations of length $n$ formed by inserting the element $n$ in one of the active sites in $\pi$. Overall, for each $n$, row $n$ of a tree will contain exactly one node for each of the permutations in $S_{n}(\Pi)$.

We will often choose the label for a node in a manner that reflects the number and/or positions of the active sites in the corresponding permutation. For instance, for our purposes, the length-1 permutation will always have two active sites, and the root of a tree will usually be given the label (2).

\section{New Wilf Equivalences}

In this section, we determine $s_{n}(123,32541)$ and $s_{n}(123,42513)$ by analyzing the structures of permutations avoiding each pair and considering their active sites, allowing us to derive generating trees and finally generating functions for the sequences $s_{n}$. Using a result of Vatter [16] and a result of Mansour and Vainshtein [10], we show that, for all $n$, $s_{n}(123,32541)=s_{n}(123,43251)$ and $s_{n}(123,42513)=s_{n}(132,34215)$ (Theorems 1 and 2$)$.

\section{$2.1 \quad S_{n}(123,32541)$}

We begin by defining three classes of permutations that avoid 123 and 32541 and proceed to find their active sites and determine the succession rules for the generating tree. By counting the number of nodes in the tree, we prove the following theorem. 


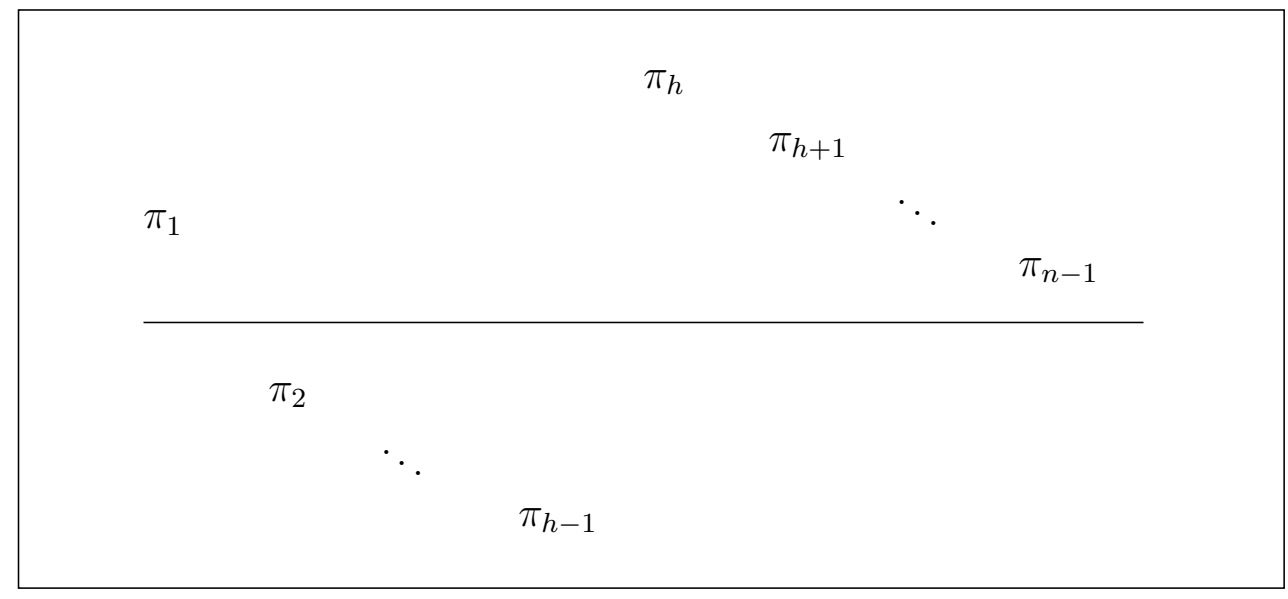

Figure 2: A permutation $\pi$ in class 1 (that is, $h>2$ and $\pi_{2}<\pi_{l}$ for all $l \geq h$ ) avoiding 123 and 32541. Inserting $n$ anywhere between $\pi_{2}$ and $\pi_{h-1}$ creates a permutation in class 2 , since $\pi_{2}>\pi_{h-1}$ and these two elements will be on opposite sides of $n$.

Theorem 1. The generating function for the sequence $s_{n}(123,32541)$ is

$$
\sum_{n=0}^{\infty} s_{n}(123,32541) x^{n}=\frac{-2 x^{5}+10 x^{4}-16 x^{3}+14 x^{2}-6 x+1}{(1-x)^{4}\left(x^{2}-3 x+1\right)}
$$

which is the same as the generating function for the sequence $s_{n}(123,43251)$, using a result of Vatter [16]. Hence $\{123,32541\}$ is Wilf-equivalent to $\{123,43251\}$.

Proof. First, note that if $\pi=\pi_{1} \pi_{2} \cdots \pi_{n-1}$ is any permutation, and inserting a new largest element $n$ into $\pi$ creates a new occurrence of some pattern $\tau$ of length $k$, then the $n$ in the new permutation must play the role of the $k$ in $\tau$, as it is larger than all other elements.

Let $\pi=\pi_{1} \pi_{2} \cdots \pi_{n-1}$ be a permutation of length $n-1$ avoiding 123 and 32541, and suppose that $\pi_{1}>\pi_{2}>\cdots>\pi_{h-1}$ is the maximal initial decreasing subsequence, in the sense that $\pi_{h-1}<\pi_{h}$ or $h=n$. The only possible active sites are those to the left of $\pi_{h}$, by 123 -avoidance. If $\pi=(n-1)(n-2) \cdots 21$, then we use the label $(n)$ for the node corresponding to $\pi$ in our generating tree; $\pi$ has $n$ active sites. If $\pi_{1}<\pi_{l}$ for all $l \geq h$, then we say that $\pi$ is in class 0 and we use the label $(h, 0)$. Here, $\pi$ has $h$ active sites, since there is no element to the right of $\pi_{h}$ to play the 1 in a new subsequence of type 32541 .

Next, if $\pi$ is not in class 0 but has $h>2$ and satisfies $\pi_{2}<\pi_{l}$ for all $l \geq h$, then we say that $\pi$ is in class 1 and we use the label $(h, 1)$; again, $\pi$ has $h$ active sites, by similar reasoning. If $h=2$, we use the label $(2,2)$, and if there exists $l \geq h$ with $\pi_{2}>\pi_{l}$, we use the label $(k, 2)$, where $k$ is the number of active sites in $\pi$. These permutations are said to be in class 2.

For permutations not in class 2 , inserting $n$ in an active site other than the leftmost one or the rightmost one creates a permutation in class 2 (see Figure 2). Moreover, this new 
permutation will only have two active sites, either because $n$ was inserted immediately after $\pi_{1}$ or because inserting $(n+1)$ anywhere between $\pi_{2}$ and $n$ in the new permutation would create a subsequence $\pi_{1} \pi_{2}(n+1) n \pi_{h-1}$ of type 32541 . Inserting $n$ before $\pi_{1}$ creates a permutation with one more active site than $\pi$ (and possibly in a different class), while inserting $n$ immediately to the left of $\pi_{h}$ creates a permutation with the same number of active sites.

For a permutation in class 2 with the label $(k, 2)$, first note that inserting $n$ at the beginning forms a permutation with label $(k+1,2)$. If there are any other active sites, then inserting $n$ in one of them creates, by an argument similar to the one in the previous paragraph, a permutation with label $(2,2)$. Thus we have our generating tree:

$$
\begin{array}{ll}
\text { Root: } & (2) \\
\text { Rules: } & (k) \rightarrow(k+1)(k, 0)(2,2)^{k-2} \\
& (k, 0) \rightarrow(k+1,1)(k, 0)(2,2)^{k-2} \\
& (k, 1) \rightarrow(k+1,2)(k, 1)(2,2)^{k-2} \\
& (k, 2) \rightarrow(k+1,2)(2,2)^{k-1} .
\end{array}
$$

Rows 1-4 of the tree are as follows:

$$
\begin{gathered}
(2) \rightarrow(3)(2,0) \rightarrow(4)(3,0)(2,0)(3,1)(2,2) \rightarrow \\
(5)(4,0)(3,0)(2,0)(4,1)(3,1)^{2}(4,2)(3,2)(2,2)^{5} .
\end{gathered}
$$

It is easy to show by induction that row $m$ contains one node labeled $(m+1)$, one node labeled $(k, 0)$ for each $k \leq m$, and, for $m \geq 3$ and each $3 \leq k \leq m$, exactly $m-k+1$ nodes labeled $(k, 1)$. Among these three types, then, there are a total of $\left(m^{2}-m+2\right) / 2$ nodes in row $m$, having a total of $\left(m^{3}+6 m^{2}-7 m+12\right) / 6$ children. Note that a node with label $(k, 2)$ has $3 k-1$ grandchildren, while any other node with $k$ children has $4 k-3$ grandchildren. Thus, counting the nodes in row $m+2$, we have

$$
\begin{aligned}
s_{m+2}(123,32541)= & 3 s_{m+1}(123,32541)-s_{m}(123,32541) \\
& +\frac{m^{3}+6 m^{2}-7 m+12}{6}-2 \frac{m^{2}-m+2}{2} \\
= & 3 s_{m+1}(123,32541)-s_{m}(123,32541)+\frac{m^{3}-m}{6} .
\end{aligned}
$$

This recurrence relation can be solved with generating functions, yielding

$$
\sum_{n=0}^{\infty} s_{n}(123,32541) x^{n}=\frac{-2 x^{5}+10 x^{4}-16 x^{3}+14 x^{2}-6 x+1}{(1-x)^{4}\left(x^{2}-3 x+1\right)}
$$

which matches the generating function for the sequence $s_{n}(123,43251)$ [16]. Because the first few values of $s_{n}(123,32541)$ are the same as the first few values of $s_{n}(123,43251)$, we have proven Theorem 1 . 


\section{$2.2 S_{n}(123,42513)$}

We begin with a few definitions in Section 2.2.1. In Section 2.2.2, we determine the active sites in permutations $\pi$ that avoid 123 and 42513, which allows us to formulate the succession rules for the generating tree in Section 2.2.3. Finally, in Section 2.2.4, we count the nodes in the tree and arrive at the following result.

Theorem 2. The generating function for the sequence $s_{n}(123,42513)$ is

$$
\sum_{n=0}^{\infty} s_{n}(123,42513) x^{n}=\frac{(1-2 x)^{2}(1-x)}{x^{4}-9 x^{3}+12 x^{2}-6 x+1},
$$

which is the same as the generating function for the sequence $s_{n}(132,34215)$, as determined by Mansour and Vainshtein [10]. Thus $\{123,42513\}$ is Wilf-equivalent to $\{132,34215\}$.

\subsubsection{Preliminary Definitions}

We begin our proof by defining certain forms of permutations $\pi$ that avoid 123 and 42513 and assigning labels to the corresponding nodes in our generating tree.

If $\pi=\pi_{1} \pi_{2} \cdots \pi_{n-1}$ avoids 123, its structure can be placed into one of two categories. If $\pi_{i}=n-1$ for some $i>1$, then we say that $\pi$ is of form 1 . We call the region to the left of $\pi_{i}$ region 1 , including the space between $\pi_{i-1}$ and $\pi_{i}$; region 1 contains $i-1$ elements.

If $\pi_{1}=n-1$, we say that $\pi$ is of form 2. In this case, we let $i$ be the largest integer such that $\pi_{i}=n-i$. If $\pi \neq(n-1)(n-2) \cdots 21$, then we define $j$ to be the integer such that $\pi_{i+j}=n-i-1$ and we call the region to the left of $\pi_{i+1}$ region 0 and the region between $\pi_{i+1}$ and $\pi_{i+j}$ region 1 . The space between $\pi_{i}$ and $\pi_{i+1}$ and the space between $\pi_{i+j-1}$ and $\pi_{i+j}$ are considered to be part of region 1 .

By 123-avoidance, the elements in regions 0 and 1 of $\pi$ are in decreasing order from left to right, and all of the sites to the right of region 1 are inactive.

Now, suppose $\pi$ avoids 123 and 42513 and is of form 1 . If $\pi$ has no occurrence of 2413 with the element playing the 2 in region 1 , then we use the label $(k, 1 \mathrm{~A})$ for the node corresponding to $\pi$, where $k$ is the number of active sites in $\pi$, and we say that $\pi$ is of form $1 A$. If $\pi$ has an occurrence of 2413 with the element playing the 2 in region 1 and $\pi$ has $k$ active sites, then we use the label $(k, 1 \mathrm{~B})$ and say that $\pi$ is of form $1 B$. Note that there must be an occurrence of 2413 with $\pi_{i}$ playing the role of the 4 , since any occurrence of 2413 must have its 4 to the right of region 1.

If $\pi$ is of form 2 , we determine its label by ignoring region 0 , finding the label $(l, 1 \mathrm{~A})$ or $(l, 1 \mathrm{~B})$ of the resulting permutation, and then assigning to $\pi$ the label $(k, 2 \mathrm{~A})$ or $(k, 2 \mathrm{~B})$, respectively, where $k$ is the number of active sites in $\pi$. We will refer to $\pi$ as being of form $2 A$ or of form $2 B$.

Finally, if $\pi=(n-1)(n-2) \cdots 21$, then we use the label $(n)$.

\subsubsection{Active Sites}

Claim 2.2(a). A permutation $\pi$ of form $2 A$ has $i+2$ active sites: the site between $\pi_{i+j-1}$ and $\pi_{i+j}$ and the leftmost $i+1$ sites in the permutation. All of the children obtained from 
$\pi$ are of form $1 A$ except the one resulting from inserting $n$ to the left of $\pi_{1}$, which is of form $2 A$.

Proof. Inserting $n$ anywhere to the left of $\pi_{i+1}$ cannot create an occurrence of 42513, as there would be no element to play the 3 . Placing $n$ to the left of $\pi_{1}$ creates a permutation of form $2 \mathrm{~A}$ with one additional element in region 0 , while placing $n$ elsewhere to the left of $\pi_{i+1}$ creates a permutation of form 1A, with anywhere from 1 to $i$ elements in region 1 .

Next, if inserting $n$ between $\pi_{i+j-1}$ and $\pi_{i+j}$ created an occurrence of 42513, then because $\pi_{i+j}$ could play neither the 1 (no element to its right is larger) nor the 3 (there needs to be a 1 between the 5 and the 3), $\pi$ would have to have contained 2413, using the elements playing the 2, 1 , and 3 in the new occurrence of 42513, plus $\pi_{i+j}$ (after the 2). This contradicts the assumption that $\pi$ is of form 2A. Note that inserting $n$ between $\pi_{i+j-1}$ and $\pi_{i+j}$ creates a permutation of form 1A; it cannot create a subsequence of type 2413, as that would imply that $\pi$ already contained 2413 (using $\pi_{i+j}$ in place of $n$ ). The new permutation formed will have $\pi_{1}=n-1, \pi_{2}=n-2, \ldots, \pi_{i}=n-i$, and $\pi_{i+1} \neq n-i-1$.

Finally, if $\pi_{i+1}$ and $\pi_{i+j-1}$ are distinct and both in region 1, then inserting $n$ anywhere between them creates an occurrence of 42513 via $\pi_{1} \pi_{i+1} n \pi_{i+j-1} \pi_{i+j}$, so there are no active sites between them.

Definition. For a permutation $\pi$ of form $1 \mathrm{~A}$ with $\pi_{i}=n-1$, let $k$ be the smallest integer such that there exists some $l>i$ for which $\pi_{1}>\pi_{l}>\pi_{k}$. If no such $k$ exists for $k<i$, then we take $k=i-1$.

Claim 2.2(b). A permutation of form $1 A$ has $k+1$ active sites: the leftmost $k$ sites and the site immediately to the left of $\pi_{i}$. One child is of form 1A, one is of form $2 A$, and the others are of form $1 B$.

Proof. First, the site immediately to the left of $\pi_{i}$ is active; if inserting $n$ there created a subsequence $\pi_{i_{1}} \pi_{i_{2}} n \pi_{i_{3}} \pi_{i_{4}}$ of type 42513 , then $\pi_{i_{1}} \pi_{i_{2}}(n-1) \pi_{i_{3}} \pi_{i_{4}}$ would be of type 42513 as well, which is a contradiction (see Figure 3 for an example). Inserting $n$ in this site creates a permutation again of form $1 \mathrm{~A}$. Next, the elements to the left of $\pi_{k}$ form a string of consecutive numbers, so all sites to the left of $\pi_{k}$ are active, as there would be no element to play the 3 in a 42513-type subsequence. Inserting $n$ to the left of $\pi_{1}$ creates a permutation of form $2 \mathrm{~A}$, while inserting it elsewhere to the left of $\pi_{k}$ creates a permutation of form $1 \mathrm{~B}$, due to the subsequence $\pi_{1} n \pi_{i-1} \pi_{i}$ of type 2413. Finally, inserting $n$ between $\pi_{k}$ and $\pi_{i-1}$ creates the type-42513 subsequence $\pi_{1} \pi_{k} n \pi_{i-1} \pi_{l}$, where $l>i$ is such that $\pi_{1}>\pi_{l}>\pi_{k}$.

Claim 2.2(c). If $\pi$ is of form $1 B$ or $2 B$, then the elements in region 1 form a string of consecutive numbers.

Proof. To show this, we shall make reference to Figure 4. Because the element playing the 2 in 2413 must be in region 1, we can ignore region 0 in our analysis. As a result, we need only consider the case in which $\pi$ is of form 1B. 


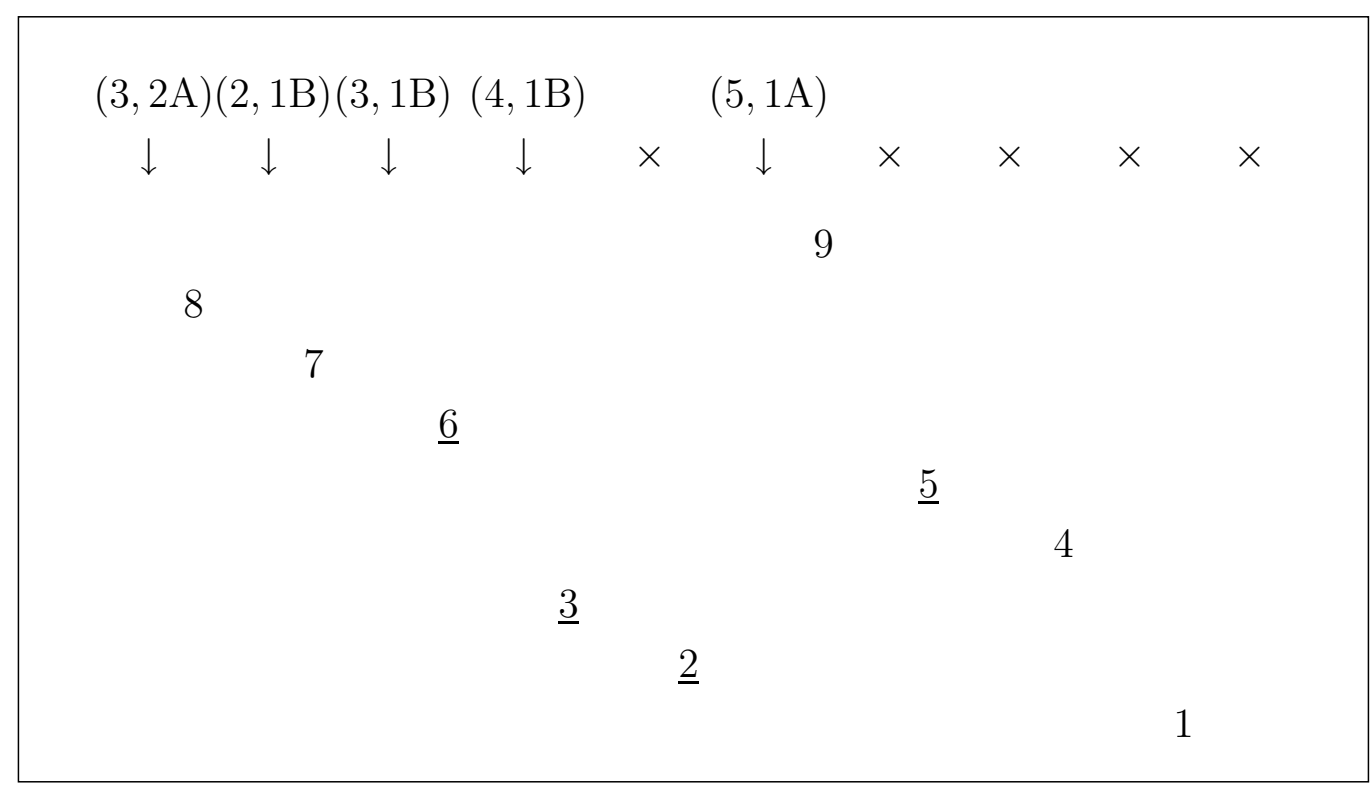

Figure 3: An illustration of the active sites in $\pi=876329541$, which has $k=4$ and is labeled $(5,1 \mathrm{~A})$. Arrows denote active sites, and the labels are shown for the length-10 permutations that would result from inserting a 10 in these sites. The underlined elements show, by 42513-avoidance, that the site between the 3 and the 2 is inactive.

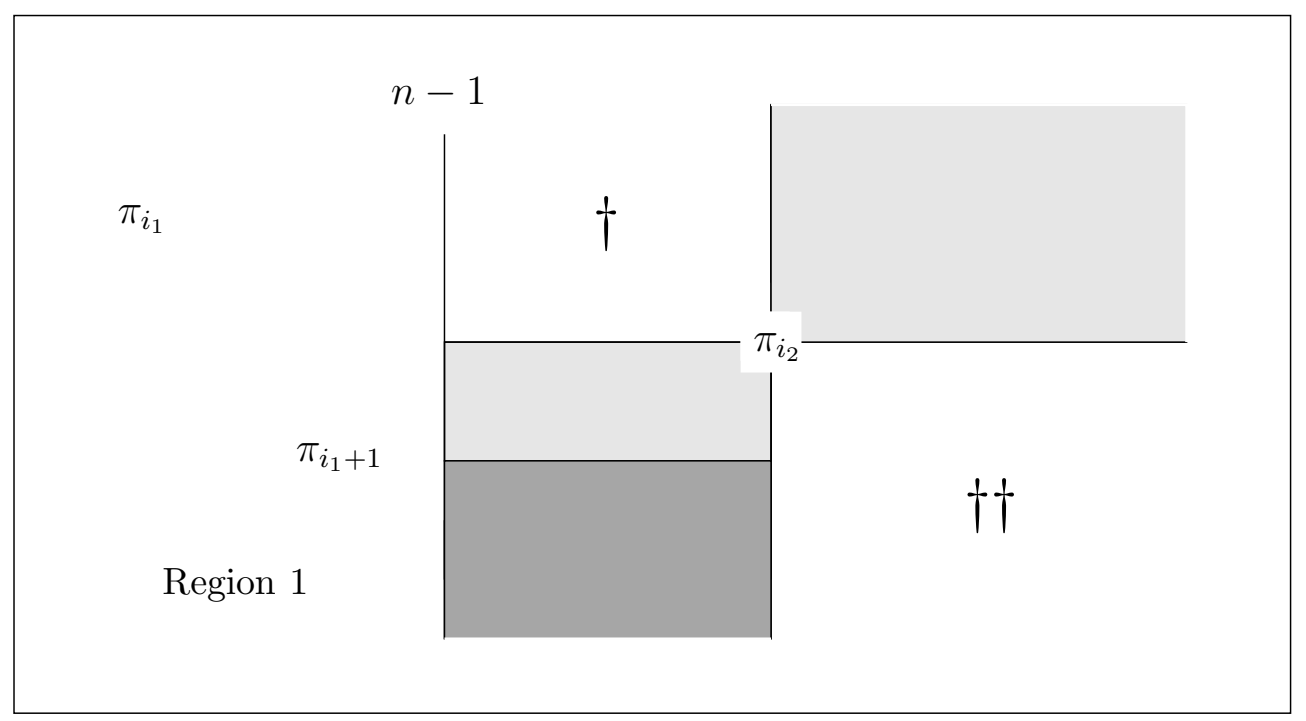

Figure 4: An element $\pi_{i_{2}}$ as shown cannot exist if the permutation contains 2413. See the proof of Claim 2.2(c). 
Suppose we had $\pi_{i_{1}}>\pi_{i_{2}}>\pi_{i_{1}+1}$ with $\pi_{i_{1}}$ and $\pi_{i_{1}+1}$ in region 1 and $\pi_{i_{2}}$ to the right of $\pi_{i}=n-1$. There must be at least one additional element to the right of $n-1$ in order for there to be an occurrence of 2413. By 123-avoidance, this element cannot be in one of the lightly shaded regions, and by 42513-avoidance, it cannot be in the darkly shaded region. Let $\pi_{l}$ be the element corresponding to the 2 in 2413 . If $\pi_{l} \geq \pi_{i_{1}}$, then there would need to be two elements in the region marked $\dagger$, in increasing order from left to right, but this would create a subsequence of type 123 using $\pi_{i_{1}+1}$. If $\pi_{l} \leq \pi_{i_{1}+1}$, then there would need to be two elements in the region marked $\dagger \dagger$, one larger than $\pi_{l}$ and one smaller, with the smaller one to the left of the larger one, but this would create a subsequence of type 42513 with $\pi_{i_{1}}, \pi_{l}$, and $n-1$. Either way, we have a contradiction.

Claim 2.2(d). The active sites in a permutation $\pi$ of form $2 B$ are precisely the leftmost $i+1$ sites. One child is of form $2 B$, and the others are of form $1 A$.

Proof. First, inserting $n$ in one of the leftmost $i+1$ sites in $\pi$ cannot create an occurrence of 42513 , because there would be no element to play the 3. Placing $n$ in any of these sites creates a permutation of form $1 \mathrm{~A}$, unless $n$ is placed in the leftmost site, in which case the new permutation is of form $2 \mathrm{~B}$.

Because we are assuming that $\pi$ contains 2413 , we may suppose that $\pi_{i+1} \pi_{i+j} \pi_{i_{3}} \pi_{i_{4}}$ is of type 2413 for some $i_{3}, i_{4}>i+j$, as the 2 in the 2413-type subsequence must be in region 1, and the elements in region 1 are consecutive numbers by Claim 2.2(c). Inserting $n$ anywhere in region 1 aside from the leftmost site, then, creates the subsequence $\pi_{1} \pi_{i+1} n \pi_{i_{3}} \pi_{i_{4}}$ of type 42513 and so is not allowed. Thus we have accounted for all of the active sites in $\pi$.

Claim 2.2(e). A permutation $\pi$ of form $1 B$ has $i$ active sites. One of its children is of form 2B, while the others are all of form $1 B$.

Proof. By Claim 2.2(c), the elements in region 1 are all consecutive numbers. Thus all sites in region 1 are active, as in Claim 2.2(d). Inserting $n$ to the left of $\pi_{1}$ creates a permutation of form $2 \mathrm{~B}$, while inserting $n$ anywhere else in region 1 creates a permutation of form 1B (using $\pi_{1}$ and the elements playing the 4, 1, and 3 in the original occurrence of 2413$)$.

\subsubsection{Succession Rules}

Having analyzed the active sites in our permutations, we are now able to write down the succession rules for the generating tree.

The only permutation $\pi$ with the label $(k)$ is $\pi=(k-1)(k-2) \cdots 21$, so it is not hard to see that

$$
(k) \rightarrow(k+1)(2,1 \mathrm{~A})(3,1 \mathrm{~A}) \cdots(k, 1 \mathrm{~A}) .
$$

For a permutation of form $2 \mathrm{~A}$, we may insert $n$ before $\pi_{1}$, somewhere else before $\pi_{i+1}$, or between $\pi_{i+j-1}$ and $\pi_{i+j}$, giving the following rule: 


$$
(i+2,2 \mathrm{~A}) \rightarrow(i+3,2 \mathrm{~A})(2,1 \mathrm{~A})(3,1 \mathrm{~A}) \cdots(i+1,1 \mathrm{~A})(i+2,1 \mathrm{~A}) .
$$

For a permutation of form $2 \mathrm{~B}$, we may place $n$ before $\pi_{1}$ or elsewhere before $\pi_{i+1}$, giving the rule

$$
(i+1,2 \mathrm{~B}) \rightarrow(i+2,2 \mathrm{~B})(2,1 \mathrm{~A})(3,1 \mathrm{~A}) \cdots(i+1,1 \mathrm{~A}) .
$$

For a permutation of form $1 \mathrm{~B}$, we may insert $n$ anywhere in region 1 , so that

$$
(i, 1 \mathrm{~B}) \rightarrow(2,2 \mathrm{~B})(2,1 \mathrm{~B})(3,1 \mathrm{~B}) \cdots(i-1,1 \mathrm{~B})(i, 1 \mathrm{~B})
$$

Lastly, for a permutation of form 1A, inserting $n$ before $\pi_{1}$ yields a permutation of form 2A, inserting $n$ between $\pi_{i-1}$ and $\pi_{i}$ yields a permutation of form 1A having the same value of $k$ as $\pi$, and inserting $n$ elsewhere before $\pi_{k}$ creates a permutation of form 1B. This leads to the rule

$$
(k+1,1 \mathrm{~A}) \rightarrow(3,2 \mathrm{~A})(k+1,1 \mathrm{~A})(2,1 \mathrm{~B})(3,1 \mathrm{~B}) \cdots(k, 1 \mathrm{~B}) .
$$

We change notation to make the tree clearer, replacing the labels $(h),(h, 2 \mathrm{~A})$, and $(h, 2 \mathrm{~B})$ with $(\mathbf{h})$ (the succession rules are functionally identical for these forms), the labels $(h, 1 \mathrm{~A})$ with $(h)$, and the labels $(h, 1 \mathrm{~B})$ with $(h, 2)$. This gives us the tree

$$
\begin{array}{ll}
\text { Root: } & (\mathbf{2}) \\
\text { Rules: } & (\mathbf{h}) \rightarrow(\mathbf{h}+\mathbf{1})(2)(3) \cdots(h) \\
& (h) \rightarrow(\mathbf{3})(2,2)(3,2) \cdots(h-1,2)(h) \\
& (h, 2) \rightarrow(\mathbf{2})(2,2)(3,2) \cdots(h, 2)
\end{array}
$$

The tree specified in this way will be referred to as Tree T.

\subsubsection{Generating Function}

The task still remains of determining $s_{n}(123,42513)$. Rows 1-6 of the generating tree are as follows:

$$
\begin{gathered}
(\mathbf{2}) \rightarrow(3)(2) \rightarrow(4)(3)(3)(2)^{2} \rightarrow(5)(4)(3)^{3}(4)(3)^{3}(2)^{4}(2,2) \rightarrow \\
(\mathbf{6})(\mathbf{5})(\mathbf{4})^{3}(\mathbf{3})^{8}(\mathbf{2})(5)(4)^{3}(3)^{8}(2)^{9}(3,2)(2,2)^{5} \rightarrow \\
(\mathbf{7})(\mathbf{6})(\mathbf{5})^{3}(\mathbf{4})^{8}(\mathbf{3})^{22}(\mathbf{2})^{6}(6)(5)^{3}(4)^{8}(3)^{21}(2)^{23}(4,2)(3,2)^{5}(2,2)^{18} .
\end{gathered}
$$

Because bold labels (4) and higher are only generated via the first rule in Tree $\mathrm{T}$, the sequence $\left\{b_{i}\right\}_{i \geq 0}=1,1,3,8,22, \ldots$, defined such that, for $m>1$, row $m$ of the tree has $b_{0}$ nodes labeled $(\mathbf{m}+\mathbf{1}), b_{1}$ nodes labeled $(\mathbf{m}), \ldots$, and $b_{m-2}$ nodes labeled $(\mathbf{3})$, is welldefined. Similarly, let $\left\{c_{i}\right\}_{i \geq 0}=1,3,8,21, \ldots$ denote the sequence whose first $m-2$ terms are the numbers of nodes labeled $(m),(m-1), \ldots,(3)$ in row $m$ (for all $m>2$ ). By considering the parent nodes of the nodes $(k)$ in some row, we can see by induction that this sequence is well-defined, as 


$$
c_{m+1}=b_{0}+b_{1}+\cdots+b_{m+1}+c_{m},
$$

regardless of the row number. Finally, we may define $\left\{d_{i}\right\}_{i \geq 0}=1,5,18, \ldots$ to be the analagous sequence for the labels $(\cdot, 2)$, including $(2,2)$. By similar reasoning, using the recurrence relation

$$
d_{m+1}=c_{0}+c_{1}+\cdots+c_{m+1}+d_{0}+d_{1}+\cdots+d_{m},
$$

we see that the sequence $\left\{d_{i}\right\}_{i \geq 0}$ is well-defined.

We need a few more facts in order to be able to count the number of nodes in the tree. First, nodes with label (2) (aside from the root) are only generated via the third rule in Tree $\mathrm{T}$, so there are $d_{0}+d_{1}+\cdots+d_{m-5}$ such nodes in row $m$ for $m \geq 5$. Write $s_{n}$ for $s_{n}(123,42513)$, with $s_{0}=1$. Every node has one child with a bold label, so, counting the bold labels in row $m+2$, we have

$$
s_{m+1}=b_{0}+b_{1}+\cdots+b_{m}+d_{0}+d_{1}+\cdots+d_{m-3} .
$$

From the first two rules in Tree $\mathrm{T}$, we see that the exponent of (2) in row $m$ is equal to the total number of bold labels in rows 1 through $m-1$, or $s_{0}+s_{1}+\cdots+s_{m-2}$. This allows us to determine the exponent of $(\mathbf{3})$ in any row and so compute that

$$
b_{m+1}=d_{0}+d_{1}+\cdots+d_{m-3}+s_{0}+s_{1}+\cdots+s_{m}+c_{0}+c_{1}+\cdots+c_{m-1} .
$$

This gives us four independent recurrence relations in the four sequences $\left\{b_{i}\right\},\left\{c_{i}\right\},\left\{d_{i}\right\}$, and $\left\{s_{i}\right\}$, which can be converted into equations in terms of the sequences' generating functions and solved simultaneously. In particular, this allows us to compute the generating function for the sequence $\left\{s_{i}\right\}$. We find that the generating function is

$$
\sum_{n=0}^{\infty} s_{n}(123,42513) x^{n}=\frac{(1-2 x)^{2}(1-x)}{x^{4}-9 x^{3}+12 x^{2}-6 x+1},
$$

which is the same as the generating fuction for the sequence $s_{n}(132,34215)$, as determined by Mansour and Vainshtein [10]. This completes the proof of Theorem 2.

\section{Further Results}

\subsection{More Generating Trees}

The fourteen generating trees in Section 3.1.2 below are for 3-5 pairs $\{\sigma, \tau\}$ that have all previously been proven to belong to the large Wilf-class of pairs with $s_{n}(\sigma, \tau)=$ $\left(3^{n-1}+1\right) / 2$, as described in Sections 1.2 and 1.5 (see [7] and [10]). We omit the full derivations of the trees and the proofs that the trees contain $\left(3^{n-1}+1\right) / 2$ nodes in row $n$. We have only been able to complete these proofs for the first nine pairs, though we 
have checked that the first several rows of the last five trees contain the proper number of nodes.

\subsubsection{Preliminaries and 132-Avoiding Permutations}

We keep in mind all observations made until this point about 123-avoiding permutations, including the definitions of regions 0 and 1 and forms 1 and 2 from Section 2.2.1.

For permutations $\pi$ avoiding 132, active sites are precisely the sites such that all elements to the left are greater than all elements to the right. We will divide a 132avoiding permutation $\pi$ into nonempty blocks of adjacent elements in such a way that the spaces between blocks are exactly the active sites of $\pi$. When we refer to sites or spaces of a permutation that avoids 132, we mean spaces between adjacent blocks. Note that inserting a new largest element $n$ into $\pi$ preserves the block structure of the elements to the right of $n$ and collapses the elements from $\pi_{1}$ though $n$ into a single block.

The following two technical claims about 132-avoiding permutations are used extensively in the derivation of the trees that follow; we present them here without proof.

Claim 3.1(a). If $\pi$ avoids 132, then each block in $\pi$ either contains 213 or has its elements in increasing order from left to right.

Claim 3.1(b). If $\pi$ avoids the two patterns 132 and $\tau=\tau_{1} \tau_{2} \tau_{3} \tau_{4} 5$, with $\tau_{4} \neq 4$, then no single block in $\pi$ contains the pattern $\tau_{1} \tau_{2} \tau_{3} \tau_{4}$.

\subsubsection{The Trees}

We now present the fourteen additional generating trees for 3-5 pairs. In writing succession rules, we use the convention that strings of labels of the form $(k)(k-1) \cdots(l)$ are ignored when $k<l$.

A few general observations can be made about the trees below. First, there are two pairs of isomorphic tress: those for $\{123,32154\}$ and $\{123,32514\}$ and those for $\{132,32451\}$ and $\{132,32415\}$. Isomorphisms, when they occur, allow us to construct bijections between the sets of permutations of length $n$ avoiding each pair, by identifying permutations corresponding to equivalent nodes in the trees. For our two cases, though, the bijections are not uniquely defined, due to the repetition of labels among the children of a single node.

There are also some pairs give rise to trees that, while not isomorphic, are quite similar: $\{132,34125\},\{132,34512\}$, and $\{123,31542\} ;\{132,45213\},\{132,42135\}$, and $\{123,42153\}$; and $\{132,52134\}$ and $\{132,21345\}$. In general, it seems as though $3-5$ pairs for which the length-5 patterns are the children of the the same length-4 pattern have similar generating trees (for instance, $\{123,32514\}$ and $\{123,32154\}$ ), whereas pairs that can be proven to be Wilf-equivalent by symmetry often have quite different trees (for instance, $\{132,32415\}$ and $\{132,42135\}$ ). It would be interesting to see if these observations could be explained systematically.
1. $\{123,32514\}$ : Root:
(2)
Rule: $\quad(k) \rightarrow(k+1)(2)(3)^{k-2}$. 
The label $(k)$ is used for a permutation $\pi$ with $k$ active sites, which are the leftmost two sites in region 1 , the rightmost site in region 1 , and all sites in region 0.
2. $\{123,32154\}$
Root:
$(2)$
Rule:
$(k) \rightarrow(k+1)(2)(3)^{k-2}$.

Again, the label $(k)$ is used for a permutation with $k$ active sites, which are the leftmost three sites in region 1 and all sites in region 0 , or all sites for the permutation $\pi=(k-1)(k-2) \cdots 21$.
3. $\{123,32415\}$ :
Root:
(2)
Rule:
$(k) \rightarrow(k+1)(k)(2)^{k-2}$.

The label $(k)$ is used for a permutation with $k$ active sites, which are the sites to the left of the leftmost block that contains 213 and the site immediately to the right of this block.
4. $\{123,32451\}$
Root:
Rule:
$(k) \rightarrow(k+1)(k)(2)^{k-2}$.

Again, the label $(k)$ is used for a permutation $\pi$ with $k$ active sites, which are now the rightmost site in $\pi$ and the sites to the left of the leftmost block that contains 213 .
5. $\{123,43215\}$
Root:
$(2)$
Rules:
$(2) \rightarrow(2)(3)$
$(3) \rightarrow(2)(3)(4)$
$(4) \rightarrow(2)(3)(4)(4)$.

Here, the label $(k)$ is used for a permutation with $k$ active sites, which are the leftmost $k$ sites in the permutation.

6. $\{123,31542\}$ :

$$
\begin{array}{ll}
\text { Root: } & (2,0) \\
\text { Rules: } & (k, l) \rightarrow(k+1, l)(0, k+1)^{l}(0, k)(0, k-1) \cdots(0,2) \text { for } k>0 \\
& (0, l) \rightarrow(1, l)(0, l)(0, l-1) \cdots(0,2) .
\end{array}
$$

The label $(k, l)$ is used for a permutation $\pi$ with $k+l$ active sites, which are the leftmost $k+l$ sites in $\pi ;(k, 0)$ is for $\pi=(k-1)(k-2) \cdots 21$, and otherwise $k$ is the number of elements in region 0 .

7. $\{132,34512\}$ :

$$
\begin{array}{ll}
\text { Root: } & (2,0) \\
\text { Rules: } & (k, l) \rightarrow(k+1, l)(0, l)^{k+1}(0, l-1)(0, l-2) \cdots(0,2) \text { for } l>0 \\
& (k, 0) \rightarrow(k+1,0)(0, k)(0, k-1) \cdots(0,2) .
\end{array}
$$

Here, if $\pi=(k-1)(k-2) \cdots 21$, then we use the label $(k, 0)$, and otherwise a label $(k, l)$ is used for a permutation with its first $k$ and last $l-2$ blocks consisting of single elements. The active sites are the leftmost $k+1$ and rightmost $l-1$ sites.

8. $\{132,34125\}$ :

$$
\begin{array}{ll}
\text { Root: } & (2,0) \\
\text { Rule: } & (k, l) \rightarrow(k+1, l)(1, k-1)(1, k-2) \cdots(1,1)(1, l)(1, l-1) \cdots(1,1) .
\end{array}
$$


The label $(k, l)$ is for a permutation whose first $k-1$ blocks are single elements, followed by a multiple-element block, followed by $l-1$ single-element blocks; the label $(k, 0)$ is for $\pi=(k-1)(k-2) \cdots 21$. The active sites are the $k+l$ leftmost sites.

$$
\begin{array}{lll}
9 .\{123,42153\}: \quad & \text { Root: } & (2,0) \\
& \text { Rules: } \quad(k, 0) \rightarrow(k+1,0)(k)(k-1) \cdots(2) \\
& (k, 2) \rightarrow(k+1,2)(k+2)(k+1) \cdots(2) \\
& (k) \rightarrow(1,2)(k)(k-1) \cdots(2) .
\end{array}
$$

If $\pi$ is of form 1, it is given the label $k$ if it has $k-1$ elements in region 1 ; the leftmost $k$ sites are all active. If $\pi$ is of form 2 , with $k$ elements in region 0 , then its leftmost $k+2$ sites are active and we use the label $(k, 2)$. The label $(k, 0)$ is used for $\pi=(k-1)(k-2) \cdots 21$.

$$
\text { 10. }\{123,42135\}: \quad \begin{array}{ll}
\text { Root: } & (2) \\
& \text { Rules: } \quad(k) \rightarrow(k+1)(k)(k-1,2)(k-2,2) \cdots(2,2) \\
& (k, 2) \rightarrow(2)(k, 2)(k-1,2) \cdots(2,2) .
\end{array}
$$

If the leftmost block in $\pi$ does not contain 213, then we use the label $(k)$ for $\pi$, where the first $k-1$ blocks in $\pi$ avoid 213 and the active sites are the first $k$ sites. If the leftmost block in $\pi$ contains 213 and the next $k-2$ blocks are single elements, then we use the label $(k, 2)$; the leftmost $k$ sites are active.

11. $\{132,45213\}$ :

$$
\begin{array}{ll}
\text { Root: } & (2,0) \\
\text { Rules: } & (k, 0) \rightarrow(k+1,0)(k, 0)(k-2,1)(k-3,1) \cdots(1,1) \\
& (k, 1) \rightarrow(k, 1)^{2}(k-1,1)(k-2,1) \cdots(1,1) .
\end{array}
$$

If $\pi$ has $k-1$ blocks, none of which contain 213 , then we use the label $(k, 0) ; \pi$ has $k$ active sites. Otherwise, if there are $k-1$ blocks to the right of the rightmost block containing 213 , then we use the label $(k, 1)$. In this case, $\pi$ has $k+1$ active sites: the rightmost $k$ spaces between blocks and the space to the left of $\pi_{1}$.

12. $\{132,21345\}$ :

$$
\begin{array}{ll}
\text { Root: } & (2,0) \\
\text { Rules: } & (k, l) \rightarrow(k+1, l)(k, l)(1,0)^{l}(1, k+l-2)(1, k+l-3) \cdots(1, l+1) \\
& \\
& (1, l) \rightarrow(2, l)(1,0)^{l} \quad(\text { for } k>2) \\
& (2, l) \rightarrow(3, l)(2, l)(1,0)^{l} .
\end{array}
$$

If the $(k+l)^{\text {th }}$ block from the left is the leftmost block in $\pi$ containing 2134 , then $\pi$ has $k+l$ active sites, which are the sites to the left of this block. If no block contains 2134, then all spaces between blocks are active. We use the label $(k, l)$ if the $k^{\text {th }}$ block from the left is the first containing 213 and the label $(k, 0)$ if no block contains 213 .

13. $\{132,52134\}$ :

$$
\begin{array}{ll}
\text { Root: } & (2,0) \\
\text { Rule: } & (k, l) \rightarrow(k+1, l)(k, l)(1, k+l-2)(1, k+l-3) \cdots(1, l+1) \\
& (0, l)(0, l-1) \cdots(0,1) .
\end{array}
$$


In this succession rule, the term $(k+1, l)$ is only included when $k>0$, and the term $(k, l)$ is only included when $k>1$. The sequence of terms $(1, k+l-2)(1, k+l-3) \cdots(1, l+1)$ is only included when $k>2$, and the sequence $(0, l)(0, l-1) \cdots(0,1)$ is only included when $l>0$.

If none of the $k+l-1$ blocks in $\pi$ contain 2134, then we use the label $(k, l)$ if the leftmost block containing 213 is the $k^{\text {th }}$ block from the left (or $(k, 0)$ if $\pi$ avoids 213); all $k+l$ sites are active. If some block contains 2134 , then we use the label $(0, l)$ if the rightmost block containing 2134 is the $l^{\text {th }}$ block from the right; the rightmost $l$ sites are active.

14. $\{132,23415\}$ :

$$
\begin{array}{ll}
\text { Root: } & (2,0) \\
\text { Rules: } & (k, l) \rightarrow(k+1, l)(1,1)^{l}(1, k+l-1)(1, k+l-2) \cdots(1, l+1) \\
& (1, l) \rightarrow(2, l)(1,1)^{l} .
\end{array}
$$

If $\pi=(k-1)(k-2) \cdots 21$, we use the label $(k, 0)$. Otherwise, suppose the $k^{\text {th }}$ block from the left is the leftmost one containing more than one element. If $\pi$ has $k+l$ blocks in total, none of which contain 123, then we use the label $(k, l)$, and all sites are active. If the $(k+l-1)^{\text {th }}$ block from the left is the leftmost one containing 123 , then there are $k+l$ active sites, and we use the label $(k, l)$.

\section{$3.2 \quad 3-6$ Pairs}

We include one generating tree for a $3-6$ pair, $\{123,362514\}$, below, along with a sketch of its derivation.

There are sixty-four 3-6 pairs that have been proven to be Wilf-equivalent with $\sum_{n=0}^{\infty} s_{n}(\cdot, \cdot) x^{n}=\left(1-4 x+3 x^{2}\right) /\left(1-5 x+6 x^{2}-x^{3}\right)$ (see [7], [9], [10], [11], and [12]). Numerical evidence shows that the only other 3-6 pair that could belong to this Wilf-class is $\{123,362514\}$. We have not been able to prove that it belongs, but from computer data, we know that $s_{n}(123,362514)$ does match the others through at least $n=11$, and the first seven rows of the tree below have been checked.

$$
\begin{array}{ll}
\text { Root: } & (2,0) \\
\text { Rules: } & (k) \rightarrow(1, k)(k)(2)^{k-2} \\
& (k, l) \rightarrow(k+1, l)(k+l)(2)(3) \cdots(k+1)(k+2)^{l-2} \text { for } l>1 \\
& (k, 0) \rightarrow(k+1,0)(2)(3) \cdots(k) .
\end{array}
$$

Let $\pi$ be a permutation avoiding 123 and 362514. If $\pi=(k-1)(k-2) \cdots 21$, we use the label $(k, 0)$, and $\pi$ has $k$ active sites.

If $\pi \neq(k-1)(k-2) \cdots 21$, then we note first that all sites in region 0 are active. If there are any inactive sites in region 1 , they must be between the elements playing the 3 and the 2 in an occurrence of 3214 having the 3 and 2 in region 1 and the 1 and 4 to the right of region 1 . We use the label $(k)$ for a permutation of form 1 with $k$ active sites and 
the label $(k, l)$ (for $l>0)$ for a permutation of form 2 with $k$ elements in region 0 and $l$ active sites in region 1 .

\section{Some Ideas for Future Work}

Eventually, it would be interesting to see if the work in this paper, perhaps extended to 3-6 pairs or other sets of patterns, could be used to help derive more general results about Wilf-equivalence. For example, perhaps relationships could be found among generating trees that would allow for a more systematic method for deriving them (see the beginning of Section 3.1.2). Also, while the Wilf-classification of 3-5 pairs is now complete, no explicit bijections are known between any two of the sets $S_{n}(\tau, \sigma)$. Finally, it would be useful to obtain a compact formula to calculate the generating function $\sum_{n=0}^{\infty} s_{n}(123, \tau) x^{n}$ for pairs $\{123, \tau\}$ with $\tau$ as general as possible, as Mansour and Vainshtein did for all pairs $\{132, \tau\}$ in $[10]$.

\section{Acknowledgements}

This research was conducted at the 2005 Summer Research Program for Undergraduates at the University of Minnesota, Duluth, under the supervision of Joseph A. Gallian. I would like to thank Joe for all his support, Ian Le for suggesting this problem and for his feedback, Phil Matchett Wood and David Arthur for their advice and encouragement, Steven Sivek for writing the computer programs I used to generate numerical data, Toufik Mansour for lending some of his knowledge of the field, and Melanie Matchett Wood and Wei Ho for reading drafts of my paper and providing many great comments. The work was supported by NSF grant DMS 0447070 and NSA grant H98230-04-1-0050.

\section{References}

[1] M. Bousquet-Mélou, Four classes of pattern-avoiding permutations under one roof: generating trees with two labels, Electronic Journal of Combinatorics 9 (2003), \#R19.

[2] F. R. K. Chung, R. L. Graham, V. E. Hoggatt, Jr., and M. Kleiman, The number of Baxter permutations, Journal of Combinatorial Theory, Series A 24 (1978), 382-394.

[3] P. Erdős and G. Szekeres, A combinatorial problem in geometry, Compositio Mathematica 2 (1935), 463-470.

[4] S. Kitaev and T. Mansour, A survey on certain pattern problems, preprint, available at http://math.haifa.ac.il/toufik/preprint.html.

[5] D. Kremer, Permutations with forbidden sequences and a generalized Schröder number, Discrete Mathematics 218 (2000), 121-130. 
[6] I. Le, Wilf classes of pairs of permutations of length 4, Electronic Journal of Combinatorics 12 (2005), \#R25.

[7] T. Mansour, 321-avoiding permutations and Chebyshev polynomials, Mathematics and computer science III; Trends in Mathematics (2004), 37-38.

[8] T. Mansour, personal communication.

[9] T. Mansour and Z. Stankova, 321-polygon-avoiding permutations and Chebyshev polynomials, Electronic Journal of Combinatorics 9(2) (2003), \#R05.

[10] T. Mansour and A. Vainshtein, Restricted 132-avoiding permutations, Advances in Applied Mathematics 26 (2001), 258-269.

[11] T. Mansour and A. Vainshtein, Layered restrictions and Chebyshev polynomials, Annals of Combinatorics 5 (2001), 451-458.

[12] T. Mansour and A. Vainshtein, Restricted permutations and Chebyshev polynomials, Séminaire Lotharingien de Combinatoire 47 (2002), Article B47c.

[13] A. Marcus and G. Tardos, Excluded permutation matrices and the Stanley-Wilf conjecture, Journal of Combinatorial Theory, Series A 107 (2004), 153-160.

[14] R. Simion and F. W. Schmidt, Restricted permutations, European Journal of Combinatorics 6 (1985), 383-406.

[15] Z. Stankova and J. West, A new class of Wilf-equivalent permutations, Journal of Algebraic Combinatorics 15 (2002), 271-290.

[16] V. Vatter, Finitely-labeled generating trees and restricted permutations, Journal of Symbolic Computation 41 (2006), 559-572.

[17] J. West, Generating trees and the Catalan and Schröder numbers, Discrete Mathematics 146 (1995), 247-262.

[18] J. West, Generating trees and forbidden sequences, Discrete Mathematics 157 (1996), 363-374. 